\title{
PhysicalBrickDatum: A Deep Physical Model for N-Dimensional Microscopy Data
}

\author{
Michael Kundmann
}

e-Metrikos, Pleasanton, California, United States

The Enabler framework provides a unified object-oriented software context for exploring, integrating, and analyzing multi-dimensional microscopy data sets [1-3]. A key component of the framework is its PhysicalBrickDatum (PBD) class [2], which implements a deep model of physically calibrated microscopy data measured along multiple dimensions. Such data sets include spectrum images (1D spectral data versus sample physical XY coordinates), in-situ time series (2D sample images versus experiment time), or ptychographic scans (2D diffraction patterns versus sample physical XY coordinates).

Recent work on Enabler has expanded the methods of PBD to enable mathematical operations and analyses between data sets having incommensurate sampling along their physical axes. Since most microscopy data are captured with detectors comprised of regularly spaced detector elements, such as CCD cameras and CMOS arrays, or via digital scan drives, this situation commonly arises when data are acquired at a variety of magnifications or dispersions or with arbitrary sampling offsets between one data series and another. Such incommensurability hinders pixel-scale quantitative comparisons of data sets and complicates the use of measured reference spectra and images as inputs to data analyses based on linear model fitting.

Figure 1 illustrates an application of PBD's inter-datum math referenced to physical data axes, rather than array indices. In this example, a simple image ratio is used to check the spatial calibration of a spectrum image (SI) against that of the reference (context) image used to specify the scan area to the SI acquisition software. If the SI calibration provided by the acquisition software is correct, then an intensity ratio between the HAADF image from the scanned subarea and the corresponding context image should be roughly constant and show no image structure. As shown at top right in Figure 1, such a ratio image quickly shows the subarea calibration to be incorrect in both scale and offset along the $\mathrm{X}$ and $\mathrm{Y}$ axes. Correcting the subarea image calibration and recomputing the ratio image yields the sort of featureless image expected. One might think such a ratio image computation to be a trivial exercise, but it is surprisingly difficult to carry out with most existing microscopy software packages because they treat data sets primarily as $\mathrm{N}$-dimensional numeric arrays. Intelligence about the meaning of a data set's physical axes and provision for resampling and interpolation, as needed for pixel-level math and quantitative comparisons, are generally missing. Even HyperSpy, the premier open-source effort towards a comprehensive package for hyper-dimensional data visualization and analysis, does not currently take account of or check for differences in the axis calibrations of data objects derived from its BaseSignal class [4].

The above is a simple case of (2D) inter-image math, but PBD has been implemented to treat all axes of an N-dimension data set on a similar basis, paving the way for arbitrary resampling and interpolation along any axis. The depth of PBD's physical model further derives from its use of Enabler's PhysicalQuantity (PQ) class to implement true intelligence about the nature of physical dimensions. PQ objects know that ' $\mathrm{nm}$ ' refers to a length and that this is compatible with and quantitatively related to ' $\mu \mathrm{m}$ ' or 'pm'. PBD inter-datum math can thus be freely applied between one image calibrated in $\mathrm{nm}$ and another in $\mu \mathrm{m}$, as long as their physical axes have some degree of overlap. Such physical know-how allows PQ to carry out physical calculations without any explicit concern for unit propagation. The internal intelligence of PQ 
handles this automatically. Only input value units and desired units on the computed results must be explicitly specified.

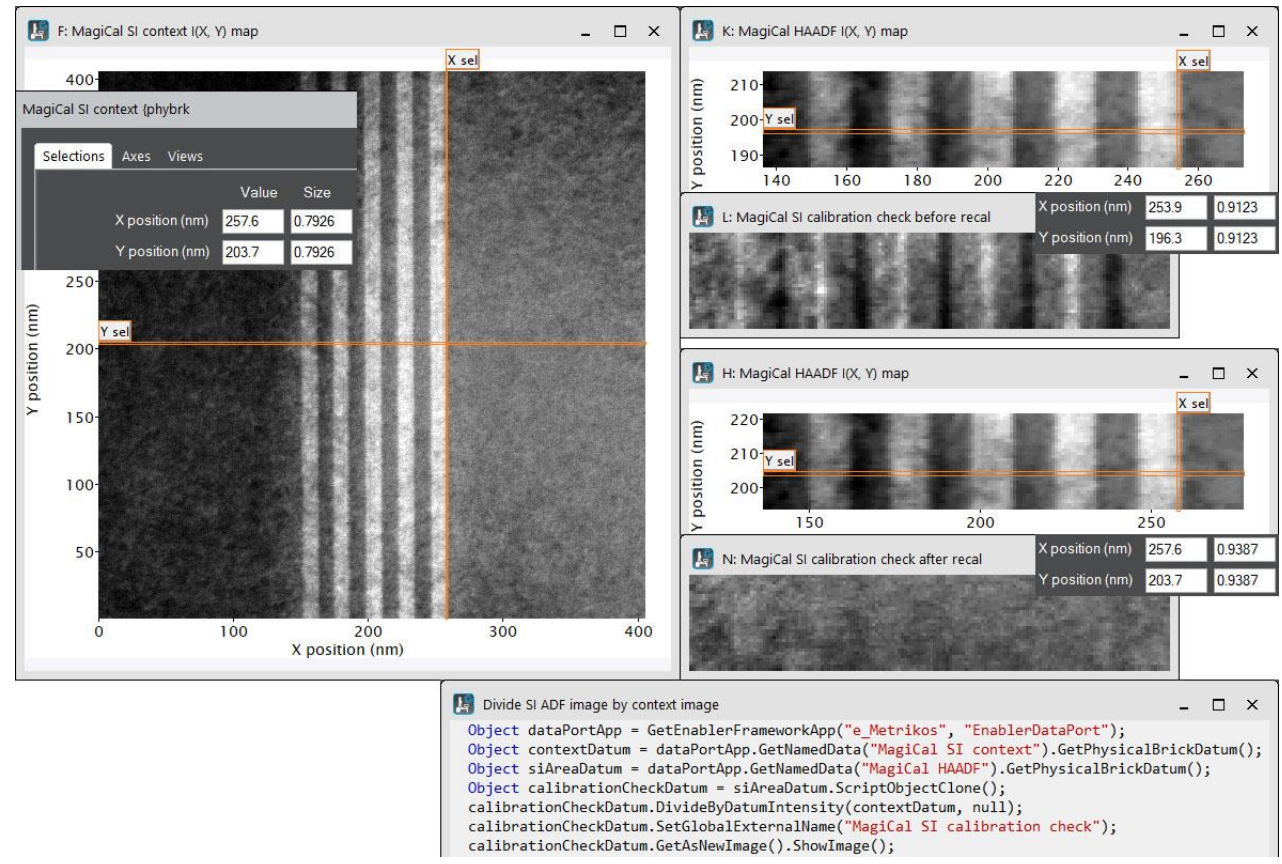

Figure 1. Example of inter-datum math based on calibrated axes, despite different sampling, scales, and sizes of the data sets. A single call to the DivideByDatumIntensity method of the PhysicalBrickDatum class provides a quick check on the accuracy of the calibration of a scanned spectrum image relative to its context image.

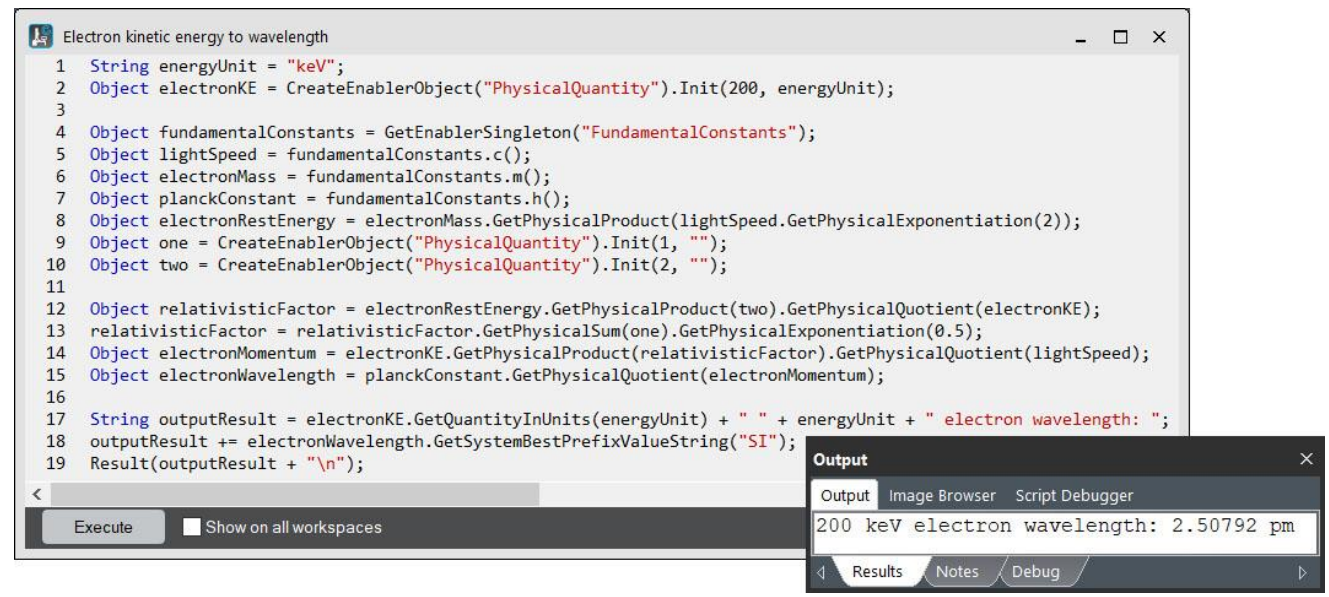

Figure 2. Script code showing use of Enabler's PhysicalQuantity class to compute electron wavelength from kinetic energy. Note framework-provided fundamental constants and automatic propagation of units from $\mathrm{keV}$ to $\mathrm{pm}$, requiring no specification of units on physical constants.

\section{References}

[1] M. Kundmann, Microscopy and Microanalysis, 25 Suppl. 2, (2019) p. 238.

[2] M. Kundmann, 2018 M\&M Meeting, late-breaking abstract and poster (un-published).

[3] M. Kundmann, Microscopy and Microanalysis, 22 Suppl. 3, (2016) p. 290.

[4] http://hyperspy.org/hyperspy-doc/current/user_guide/tools.html\#signal-operations 